\title{
Enhanced transmission and beaming of light via photonic crystal surface modes
}

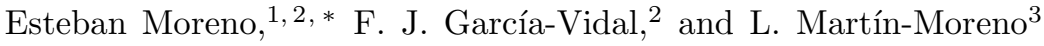 \\ ${ }^{1}$ Laboratory for Electromagnetic Fields and Microwave Electronics, \\ Swiss Federal Institute of Technology, ETH-Zentrum, CH-8092 Zurich, Switzerland \\ ${ }^{2}$ Departamento de Física Teórica de la Materia Condensada, \\ Universidad Autónoma de Madrid, E-28049 Madrid, Spain \\ ${ }^{3}$ Departamento de Física de la Materia Condensada, \\ Universidad de Zaragoza-CSIC, E-50009 Zaragoza, Spain
}

\begin{abstract}
Surface modes are generally believed to be an undesirable feature of finite photonic crystals (PC), unlike point or line defect modes. However, it is possible to make the surface mode radiate by appropriate corrugation of the PC interface. In this paper we show theoretically that the coherent action of these surface indentations can be engineered to collimate within a few degrees the light exiting a PC waveguide, or to funnel light coming from free space into the waveguide.
\end{abstract}

PACS numbers: 42.70.Qs, 78.20.Ci, 42.79.Ag

The proposal of photonic crystals (PC) 1.2 in dielectric materials has stimulated the search of photonic band gap effects in periodic metallic structures $3.4,5,6.7,8,9.10$. These latter structures have shown remarkable properties, such as extraordinary optical transmission both through subwavelength hole arrays ${ }^{11}$ and single apertures ${ }^{12}$, and beaming of light from single apertures flanked by periodic surface corrugations ${ }^{13}$. The root of all these phenomena lies in the existence of surface electromagnetic (EM) modes at metal-dielectric interfaces ${ }^{14.15 .16}$. PCs can also support surface EM modes for appropriate crystal termination surfaces ${ }^{17,18,19}$. Additionally, for frequencies within the band gap, the PC behaves as a mirror for incoming EM radiation. These two facts suggest that the cited optical phenomena found in metals could have counterparts in PC systems. However, this analogy is not straightforward as the field penetration inside PCs (of the order of one wavelength) is much larger than the skin depth in good metals in the optical regime, which could strongly modify the interference phenomena we are referring to. In this paper we explore the possibility of exporting some of the results found in metallic structures to dielectric PCs. We will show that, through appropriate corrugation of the $\mathrm{PC}$ surface, it is possible to obtain both enhanced transmission through slits in PC slabs, and strong beaming of light coming out of a PC waveguide.

Let us first discuss the basics of the beaming phenomena in metallic systems, considering the simpler onedimensional case of a guided mode coming out of one single slit flanked by a finite array of grooves 15 . The grooves act as centers that re-scatter the EM radiation diffracted by the slit exit. For certain resonant conditions a leaky surface EM wave develops, which emits into vacuum as it propagates along the surface. The phase accumulated during this surface propagation can be compensated, after emission, by optical paths in free space. Therefore, for certain angles, radiation coming from all indentations interferes constructively, leading to beaming. This mechanism suggests the strategy shown in this paper in order to pursue the analogy: first a waveguide in a $\mathrm{PC}$ is constructed and then, by modifying the PCvacuum interface, a surface EM mode is created. Finally, in order to achieve the desired beaming effect, this mode is connected to the radiative modes by adding a periodic modulation to the PC surface. Additionally, these surface leaky modes could also be used to funnel light from free space into the waveguide, therefore enhancing the transmission, as has been previously demonstrated in single apertures surrounded by a periodic corrugation in a metallic film 16 .

In this paper, for proof of principle purposes, we concentrate in two-dimensional PCs. As photonic band gap (PBG) system, we consider a square lattice of cylinders in vacuum. We choose cylinder radius $r=0.18 a$ ( $a$ being the lattice parameter) and dielectric constant of the cylinders $\epsilon_{\mathrm{r}}=11.56$ (we take $\epsilon_{\mathrm{r}}$ for GaAs at a wavelength of $1.5 \mu \mathrm{m}$ as a representative value). For this set of parameters the system presents, for E-polarization (electric field pointing along the cylinders axis), a frequency gap for reduced frequencies $\omega a / 2 \pi c$ in the range $(0.30,0.44)$, where $c$ is the speed of light. All results presented are for E-polarization and have been obtained with the multiple multipole (MMP) method 20 . The computational technique to treat $\mathrm{PC}$ waveguide discontinuities within the MMP method is thoroughly described in Refs. 2122. A waveguide is constructed by removing one row of cylinders in the $z$-direction (see inset of Fig. 2). For most frequencies in the PBG this waveguide supports one propagating mode. The PC is infinitely extended towards $z \rightarrow-\infty$ but, in order to study the coupling of the waveguide mode into free space, we terminate the $\mathrm{PC}$ in a set of cylinders parallel to the $x$-direction (in the coordinate system we have chosen, the center of these last cylinders is at $z=7.5 a$ ). This regular $\mathrm{PC}$ termination does not support surface states 18 . Fig. 1(a) shows the real space electric field amplitude when a guided mode with frequency $\omega_{0}=0.408(2 \pi c / a)$, launched at $z \rightarrow-\infty$, is diffracted by the waveguide exit. The radiated electric field shows a broad angular distribution, as corresponds to an aperture of dimensions comparable to the wavelength (the wavelength corresponding to 

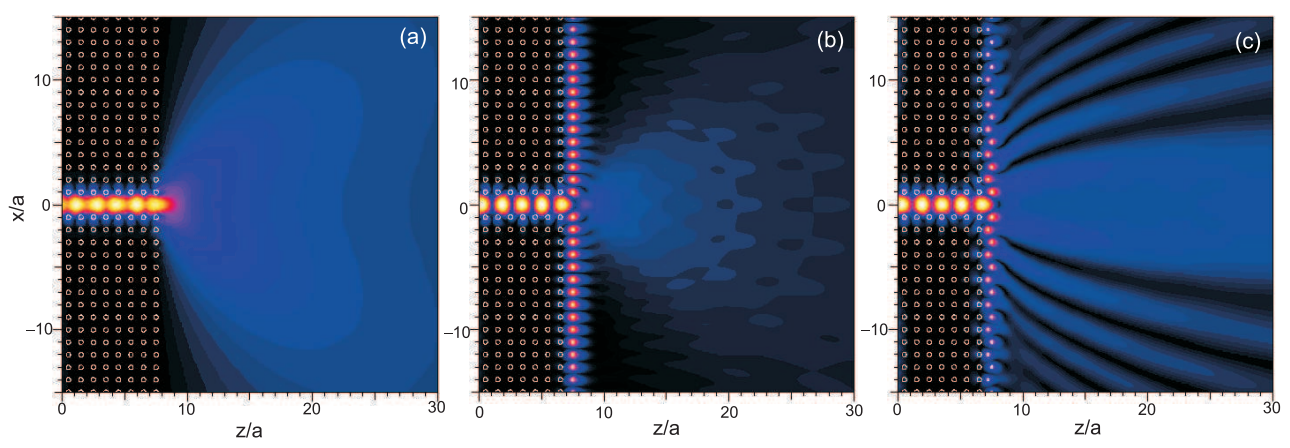

FIG. 1: Electric field amplitude $|E(z, x)|$ radiated out of a PC waveguide for various crystal interfaces. The input field is a guided mode coming from the left. (a) Standard interface (no surface modes allowed), (b) the cylinders at the interface have smaller radius than those in the PC bulk (one non-radiative surface mode supported), and (c) same as in (b) but with additional periodic modulation of the interface cylinders (one leaky surface mode supported). The color scales are linear and extend from black (0) to white (maximum), the units are arbitrary.

$\omega_{0}$ is $\left.\lambda_{0}=2.45 a\right)$. This is more clearly seen in Fig. 2 (black line), rendering the far-field radial component of the Poynting vector, $S_{r}$, as a function of azimuthal angle, $\theta$. In order to create a surface state, one possibility would be to cut the outer part of the interface cylinders, thus leaving one monolayer of hemicylinders $\frac{18}{}$. The origin of this surface state is that a mode that would be in the dielectric band for complete cylinders shifts its energy up when part of the dielectric is removed ${ }^{17}$. In this work the surface state is induced in a similar manner by decreasing the radius $r_{\mathrm{i}}$ of the interface monolayer of cylinders, which is a more convenient system to treat by the MMP method. For the ulterior illustration of beaming in the

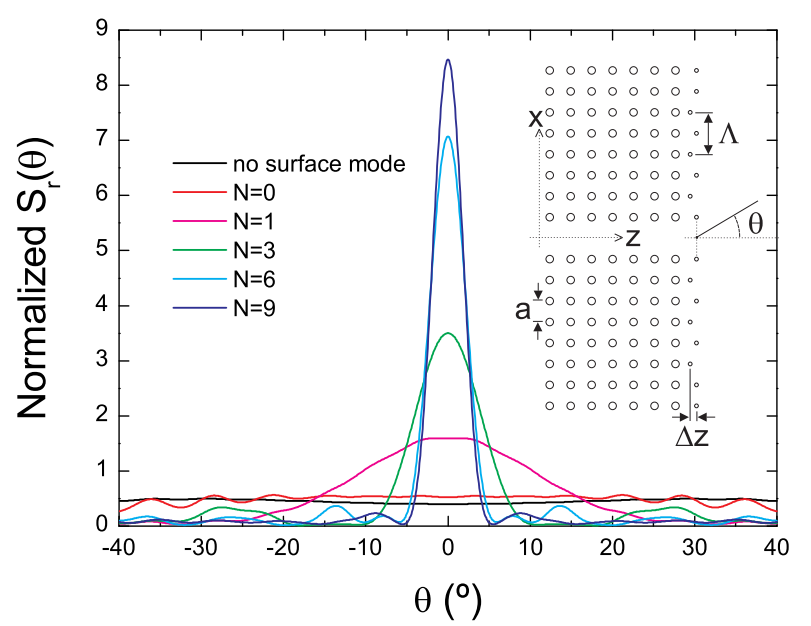

FIG. 2: Far-field radial component of the Poynting vector, $S_{r}$, radiated out of a $\mathrm{PC}$ waveguide as a function of azimuthal angle, $\theta$. In all cases, the integral in $\theta$ of the angular transmission distribution, $r S_{r}(\theta)$, is normalized to unit. The black curve corresponds to a standard interface, whereas the remaining curves correspond to an interface with cylinders radius $r_{\mathrm{i}}=0.09 a$. $N$ stands for the number of corrugations in the modulated surface (see text). Inset: scheme of the considered structures. forward direction, it is convenient to choose $r_{\mathrm{i}}$ in such a way that a surface mode appears with a modal wavelength $\lambda_{\mathrm{sm}}=2 a$. For the chosen $\omega_{0}$ this occurs approximately for $r_{\mathrm{i}}=0.09 a$. Fig. 团 b) renders a similar plot to Fig. 11a) but, this time, all cylinders in the interface monolayer have that particular $r_{\mathrm{i}}$. The presence of a surface mode being fed by the waveguide is clear in Fig. 1(b), but as this mode does not couple to radiative modes, the radiated power again spreads in all directions as corresponds to an aperture size of the order of $\lambda_{0}$. This point is corroborated by Fig. 2 (red curve) showing an almost uniform $S_{r}(\theta)$ for the case considered. In order to alter the radiation pattern, the surface mode should couple to the continuum of radiative modes. This can be achieved by introducing a periodic modulation to the PC surface. In this way the surface state can "pick up" a reciprocal lattice vector of the superimposed lattice and connect to waves inside the light cone. Out of the different possibilities for this modulation, we present here results for a simple choice with a corrugation period $\Lambda=2 a$ : at either side of the waveguide exit, the cylinders at the PC-vacuum interface in the first $N$ even positions (we order the cylinders consecutively according to their distance to the waveguide) are shifted a distance $\Delta z$ along the $z$-axis. Fig. प(c) renders the real space electric field amplitude for $\Delta z=-0.3 a$ and $N=9$. This plot is radically different from the one obtained for systems without surface mode or with a non-radiative surface mode, distinctly showing the re-emission process. The beaming characteristics are clearly seen in the corresponding farfield transmission angular distribution shown in Fig. 2 for $N=1,3,6,9$. These curves present a strong redistribution of the transmitted radiation into the forward direction, with a beam angular width of a few degrees.

In the corrugated metal case, a focus in the Fresnel regime was found associated to the beaming in the farfield region ${ }^{23}$. This is also the situation in the present case. Fig. 31(a) shows the real space dependence of the Poynting vector modulus, $S$, for a modulated surface with $N=3$, where already the presence of a colli- 

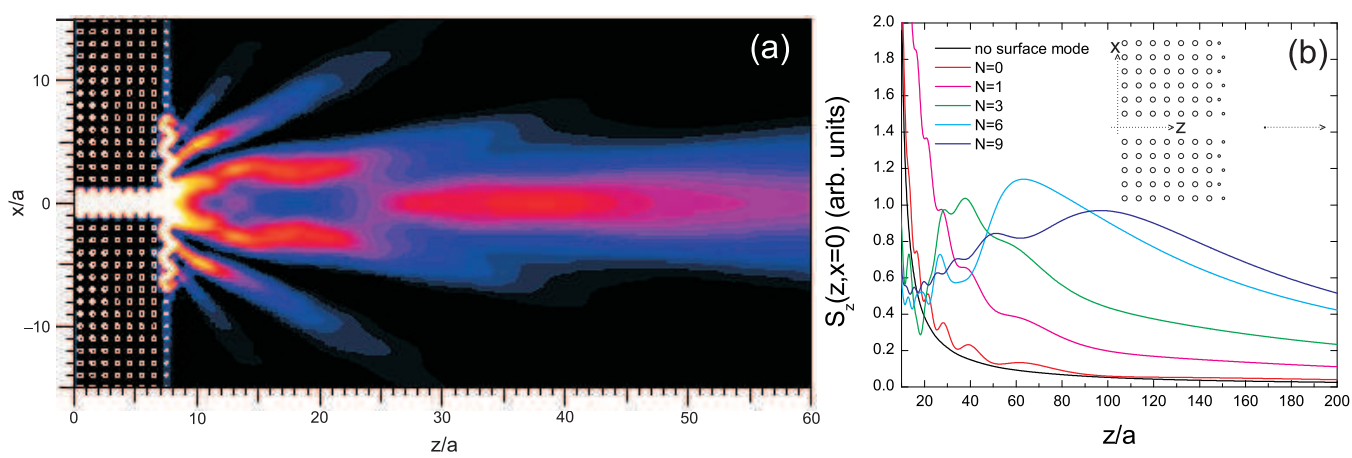

FIG. 3: (a) Poynting field modulus $S(z, x)$ radiated out of a PC waveguide. The PC-vacuum interface supports one surface mode which radiates due to a periodic modulation of the surface (number of corrugations $N=3$ ) leading to beaming and lensing. (b) Poynting field $S_{z}(z, x=0)$ along the waveguide axis (the dotted line in the inset) for increasing number of corrugations at the interface.

mated beam and a focus is apparent. In similar way to the metallic counterpart, the focus characteristics depend on the number of periods of modulation in the surface. As illustration, Fig. [3(b) shows $S_{z}(z, x=0)$ for $N=0,1,3,6,9$. The maxima of these curves provide the focal distance of the, very elongated, focus. In order to further assign these effects (beaming and lensing) to the existence of a leaky surface mode, and to rule out that they are only due to the modification of the waveguide opening induced by the corrugation, the $x$-component of the Poynting vector, $S_{x}$, can be scrutinized along the interface. Fig. 4 shows $S_{x}(x, z=7.5 a)$ for the cases of no surface mode, surface mode $(N=0)$, and surface leaky mode for $N=3,9$. For $N=0$, after an initial depen-

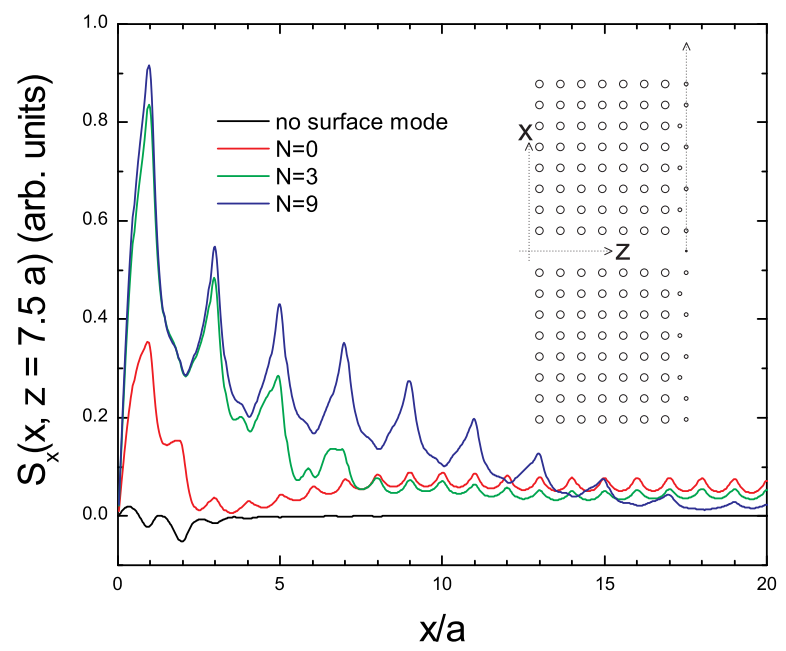

FIG. 4: Poynting field $S_{x}(x, z=7.5 a)$ along the PC-vacuum interface (dotted line in the inset). The black curve corresponds to a standard interface (no surface mode supported), whereas the remaining curves correspond to an interface with cylinders radius $r_{\mathrm{i}}=0.09 a$ (one surface mode supported). $N$ stands for the number of corrugations in the modulated surface. dence on $x$ for small distances to the waveguide (due to the coupling between the waveguide mode and the surface mode), the modal carried power remains constant, as expected for the non-radiative surface mode present in the system. On the contrary, cases with $N \neq 0$ show that the field decreases as the mode travels along the surface, due to the radiation losses responsible for the beaming and lensing phenomena. Notice that the case with a surface termination that does not support surface modes or resonances, presents an almost negligible intensity along the surface. It is worth stressing here that, although we have chosen a favorable range of parameters in order to show that the cited effects exist, no attempt has been made in order to optimize the structures. This is already apparent in Figs. 1 b, c), where the strong electric field modulation inside the waveguide is due to a high reflection [R=52.3\% and $R=24.5 \%$ in (b) and (c), respectively] caused by the large impedance mismatch between waveguide and free space regions. This reflection can be largely modified by very local changes of the dielectric structure at the opening (such as tapering ${ }^{22}$ ). Similarly, the coupling between waveguide mode and surface resonance surely has a strong influence on the transmission angular distributions. Which geometry is optimal is an interesting question that, however, falls outside the scope of this paper.

Beaming at other angles is also possible. For instance, for the chosen frequency $\omega_{0}$, shifting normally to the surface one every three cylinders $(\Lambda=3 a)$, results in two collimated beams at $\theta= \pm 24^{\circ}$. For the system presented in this paper, coupling to a surface state with an in-plane wave vector different from $2 \pi / \Lambda$ (which would occur at certain frequencies different from $\omega_{0}$ ) would also result in beaming at $\theta \neq 0^{\circ}$.

As previously said, another interesting effect found in metallic structures is that a structure presenting beaming when corrugated in the exit surface, is capable of presenting enhanced transmission when that corrugation is induced in the input surface. Fig. 5 ] shows the transmittance through a waveguide in a PC slab when illuminated 


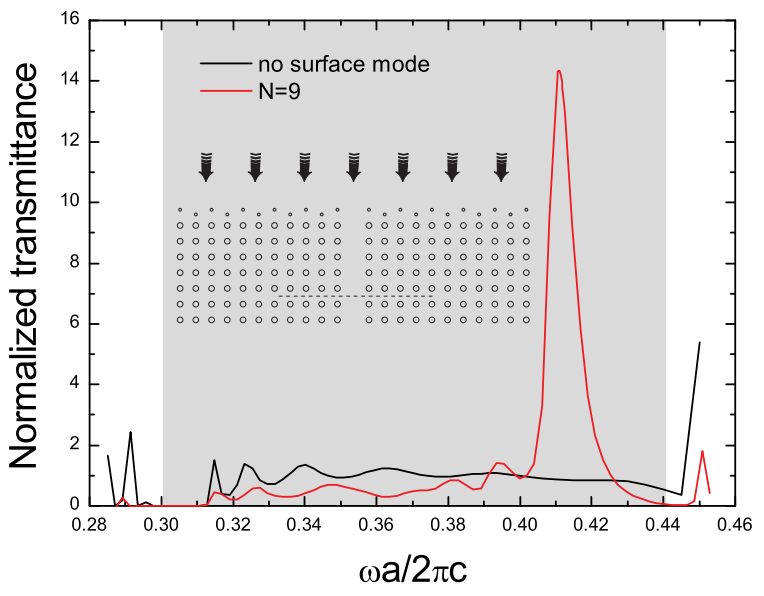

FIG. 5: Normalized transmittance through a waveguide in a PC slab. The gray shading represents the PBG. Black curve: standard interface (no surface mode supported); red curve: interface with monolayer of cylinders with radius $r_{\mathrm{i}}=0.09 a$ (one leaky surface mode supported) and corrugated with $N=9$ indentations. The considered structure is finite [slab thickness: 8 layers, lateral extension: $40 a$ (with the central cylinder line removed to create the waveguide)]. The employed normalization is explained in the text.

by a normally incident plane wave. The black curve corresponds to a regular unstructured interface [same as in Fig. 1(a)], whereas the red curve corresponds to the corrugated interface shown in Fig. 1(c). The transmittance has been normalized to the power that impinges onto a length $a$ (i.e., roughly the waveguide cross section). In both curves rendered in Fig. 囵 transmission outside the PBG is due to the EM field permeating through the crystal, while the region of transmission of order unity inside the gap is due to propagation through the waveguide. Interestingly, Fig. 5 shows a large resonant transmission inside the gap for the $\mathrm{PC}$ with a modulated surface. The resonant frequency is the same that was previously obtained for beaming of light out of a waveguide. Notice that, even in this case without full optimization of the geometry, the energy flux collected into the waveguide corresponds to the one impinging in a cross section around 14a. This effective cross section could be increased, for instance, by considering a shallower corrugation with a larger lateral size. Unlike the analog situation in metallic structures, here the system is lossless, and therefore dissipation does not set a limit to the possible device lateral size.

To summarize, in this paper we have demonstrated the use of surface leaky modes, appearing in PCs under appropriate conditions, to collimate EM radiation out of $\mathrm{PC}$ waveguides. Very narrow beams with angular distributions of a few degrees can be achieved by modulating the $\mathrm{PC}$ exit surface. Furthermore, these surface resonances may also be used for injecting light efficiently into these waveguides. This enhanced transmission property could have interesting applications in the efficient coupling between conventional optical fibers and PC-based waveguides. (Funding by the Swiss National Science Foundation/NCCR Quantum Photonics and financial support by the Spanish MCyT under contracts MAT2002-01534 and MAT2002-00139 are gratefully acknowledged).

* Electronic address: esteban.moreno@uam.es

1 E. Yablonovitch, Phys. Rev. Lett. 58, 2059 (1987).

2 S. John, Phys. Rev. Lett. 58, 2486 (1987).

3 A. R. McGurn and A. A. Maradudin, Phys. Rev. B 48, 17576 (1993).

${ }^{4}$ D. R. Smith et al., Appl. Phys. Lett. 65, 645 (1994).

5 S. C. Kitson, W. L. Barnes, and J. R. Sambles, Phys. Rev. Lett. 77, 2670 (1996).

${ }^{6}$ F. J. Garcia-Vidal and J. B. Pendry, Phys. Rev. Lett. 77, 1163 (1996).

7 S. Fan, P. R. Villeneuve, and J. D. Joannopoulos, Phys. Rev. B, 5411245 (1996).

8 D. F. Sievenpiper et al., Phys. Rev. Lett. 80, 2829 (1998).

9 S. I. Bozhevolnyi et al., Phys. Rev. Lett. 86, 3008 (2001).

10 J. G. Fleming et al., Nature (London) 417, 52 (2002).

11 T. W. Ebbesen et al., Nature (London) 391, 667 (1998).

12 T. Thio et al., Opt. Lett. 26, 1972 (2001).

13 H. J. Lezec et al., Science 297, 820 (2002).

14 L. Martin-Moreno et al., Phys. Rev. Lett. 86, 1114 (2001).

15 L. Martin-Moreno et al., Phys. Rev. Lett. 90, 167401 (2003).

16 F. J. Garcia-Vidal et al., Phys. Rev. Lett. 90, 213901 (2003).

17 R. D. Meade et al., Phys. Rev. B 44, 10961 (1991).

18 J. N. Winn, R. D. Meade, and J. D. Joannopoulos, J. Mod. Opt. 41, 257 (1994).

19 F. Ramos-Mendieta and P. Halevi, Phys. Rev. B 59, 15112 (1999).

20 Ch. Hafner, Post-modern electromagnetics, John Wiley and Sons, Chichester (1999).

21 E. Moreno, D. Erni, and Ch. Hafner, Phys. Rev. B 65, 155120 (2002).

22 E. Moreno, D. Erni, and Ch. Hafner, Phys. Rev. E 66, 036618 (2002).

${ }^{23}$ F. J. Garcia-Vidal et al., Appl. Phys. Lett. (in press). 